Journal of Case Reports 2020;10(3):197-200

\title{
Large Schwannoma of the Tibial Nerve
}

\author{
Swagat Mahapatra, Pankaj Aggarwal, Harsh Pratap Singh \\ Department of Orthopaedics, Dr. Ram Manohar Lohia Institute of Medical Sciences, Lucknow, Uttar Pradesh, India.
}

\begin{abstract}
Corresponding Author:
Dr Swagat Mahapatra

Email: drswagat@gmail.com

This is an Open Access article distributed under the terms of the Creative Commons Attribution License (creativecommons.org/ licenses/by/3.0).
\end{abstract}

$\begin{array}{lll}\text { Received } & : & \text { March 8, 2020 } \\ \text { Accepted } & : & \text { July 31, 2020 } \\ \text { Published } & : & \text { September 25, } 2020\end{array}$

\begin{abstract}
Background: Schwannomas are very common, benign, encapsulated tumors of the peripheral nerves. They rarely emerge from the posterior tibial nerve. Case Report: Here we report a case of an unusual schwannoma measuring $10 \times 5 \times 4 \mathrm{~cm}$ in a 24 -year-old man. The mass was painful, and Tinel's sign was positive. Magnetic resonance imaging revealed that it originated from the posterior tibial nerve. En bloc resection of the tumor was performed. Histology revealed peripheral nerve schwannoma. Removal of tumor made patient pain free after surgery. Conclusion: A high index of suspicion with meticulous clinical examination is necessary for timely diagnosis of schwannoma.
\end{abstract}

Keywords: Peripheral Nervous System Neoplasms, Radiculopathy, Tibial nerve, Magnetic Resonance Imaging.

\section{Introduction}

A schwannoma is a non-invasive, well encapsulated benign tumor of the peripheral nerve sheath. The cells of origin of schwannoma's are Schwann cells of primitive neuroectoderm [1,2]. Five percent of all soft tissue tumors are schwannomas [3]. They have no gender predilection, vary in size from $1-3 \mathrm{~cm}$, and they characteristically occur between the second and fifth decade [4-6]. Schwannomas frequently arise in the cephalic and cervical region involving the brachial plexus and spinal nerves. The upper and lower limbs are involved less often [7]. These tumors may be managed conservatively unless symptoms or neurological deficit emerge for which surgical excision is done. We report a case of schwannoma located in the tibial nerve.

\section{Case Report}

A 24-year-old, young adult male presented with a protracted history of left leg pain and paresthesia that was increasing over past one year. There was no history of any relevant trauma, low back pain and radiation. General physical examination and spine examination revealed no abnormality. On physical examination of the knee, a palpable mass of size approximately $10 \times 5 \times 4 \mathrm{~cm}$ was found on the proximal one third of the posterior surface of leg. The mass was firm and tender on palpation. Tinel's

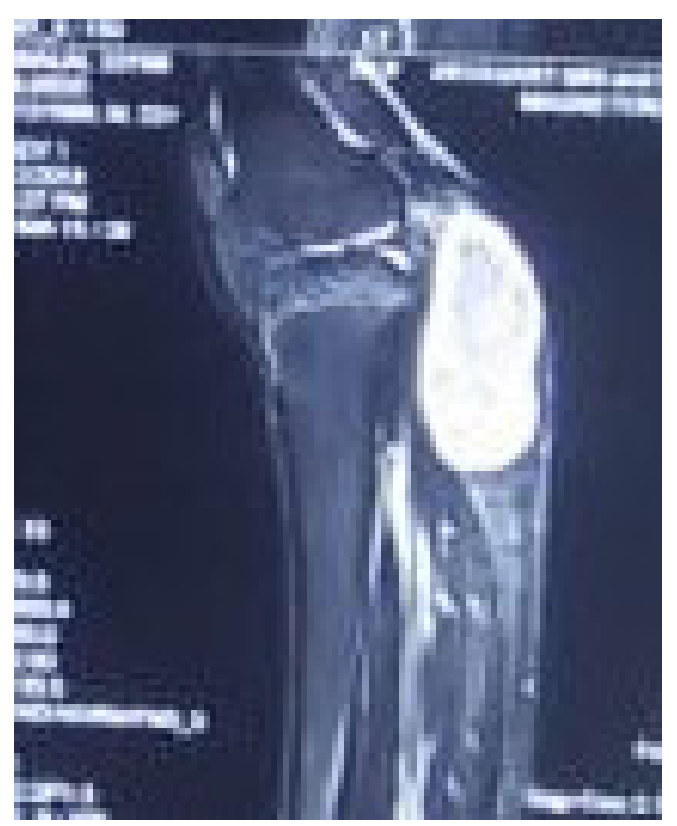

Fig.1: MRI showing schwannoma of the tibial nerve in the popliteal fossa and proximal leg. 
sign was positive over the mass. Knee flexion was limited and painful at seventy degrees. There was no objective motor and sensory deficit.

Radiographs revealed no bony abnormality but MRI revealed a well circumscribed $9.0 \times 5.0 \times 4.5$ $\mathrm{cm}$ mass in popliteal fossa and proximal leg arising from the posterior tibial nerve. The mass was eccentric as per scans and gave high index of suspicion for a schwannoma. A soft tissue needle biopsy was done and histologic examination favored the diagnosis of schwannoma. In due course patient underwent surgery for resection of the mass. We planned neuromonitoring for confirming the status of the nerve intraoperatively. Under general anesthesia, and tourniquet control, the patient was placed in prone position and a lazy $\mathrm{S}$ incision was made over left popliteal fossa. All neurovascular structures were identified and protected. The sciatic nerve was identified and further traced distally to find the bifurcation of common peroneal nerve and posterior tibial nerve. The posterior tibial nerve was further traced down to trace a large oval mass of size approximately $9 \times 5 \times 4 \mathrm{~cm}$ arising from the nerve. Further microdissection under magnification was done to protect all nerve fibers and the mass was exposed. The mass was excised without any damage to the proximal and distal branches of the nerve. Post-operatively left lower limb was immobilized in above knee pop slab for 2 weeks following which suture removal was done and range of motion and weight bearing were allowed. Clinical examination at 6 weeks, 10 weeks, 16 weeks and 6 months revealed no neurological deficit.

\section{Discussion}

Benign nerve sheath tumors can be categorized into schwannomas and neurofibromas. Schwannomas are usually solitary and well encapsulated similar to our case. Schwannoma has been reported to be eccentrically located and does not involve the main nerve [8]. Schwannoma tends to displace the nerve fibers peripherally in contrast to neurofibromas,

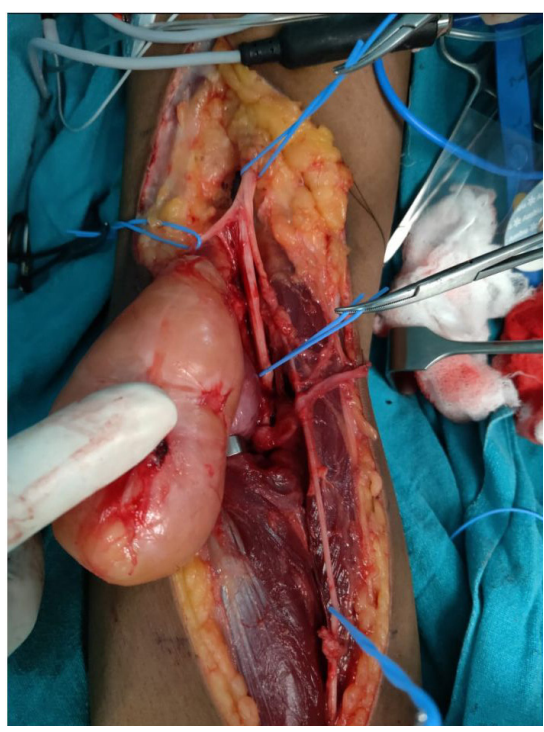

Fig.2: Intra-operative dissection of the tumor.

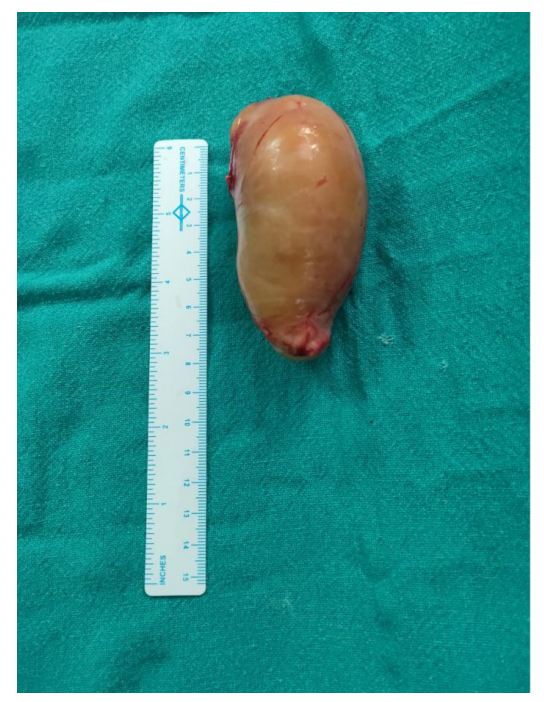

Fig.3: Dissected specimen.

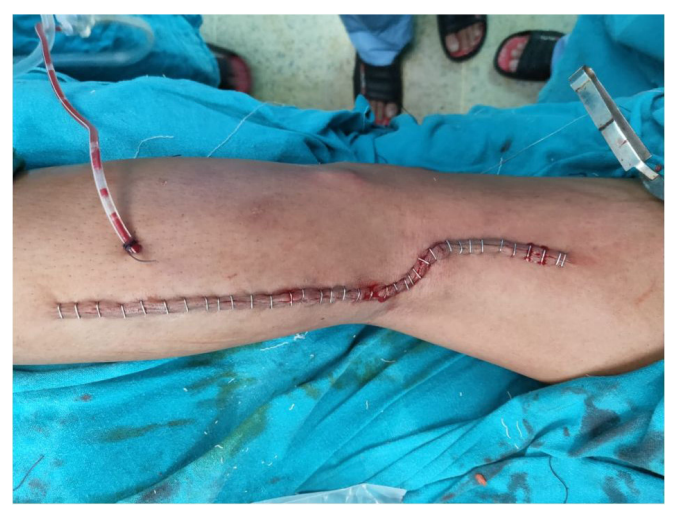

Fig.4: Immediate post-operative closure. 
which usually grow within the nerves and infiltrate them and hence require partial or complete removal of the nerve producing neurological deficit. Hence it is very important to get a preoperative tissue diagnosis for explaining the exact nature and complications of surgery. Schwannoma is a slow-growing mass and often is asymptomatic. Schwannoma presents as a palpable mass with local pain and paresthesia. Schwannomas most commonly occur in the peripheral nerve plexuses and spinal nerves. The lower and upper limb nerves are affected less often. In the upper extremity, ulnar nerve is most commonly involved while in the lower extremity tibial nerve is most often involved [9].

The diagnosis of tibial nerve schwannoma in this case was tricky and delayed due to its unusual appearance in the lower extremity, which is not a common site. Also, late appearance of the focal swelling of this slow growing tumor coupled with minimal clinical signs mimicking a radiculopathy contributed to the delay. Importance has to be given to meticulous clinical examination along with imaging modalities for early diagnosis and management of such cases. We have used portable nerve stimulator intra-operatively to check the functioning of the intact nerve after dissecting out the tumor. This has not been described in recent studies. The loupe magnification and microinstruments during surgery give a better clinical outcome by facilitating meticulous dissection. This point has also been stressed by Rajasekaran ET et al. [10] and Kim ET et al. [11]. Complete resection using magnification, nerve stimulators and good microsurgical dissection should be attempted for better prognosis and decrease recurrence rates.

\section{Conclusion}

Schwannomas arising from posterior ibial nerve are rare and a high index of suspicion with meticulous clinical examination is necessary for its timely diagnosis. Preoperative radiological and tissue diagnosis will also help explain the prognosis of

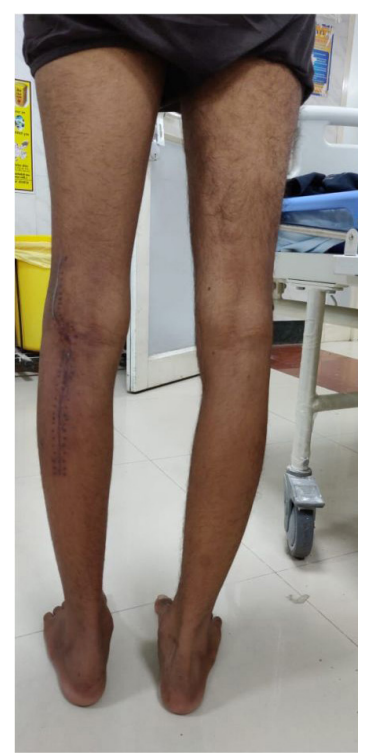

Fig.5: Post-operative scar at 10 weeks with full knee extension.

treatment. Finally use of magnification loupes, nerve stimulator and micro-instruments during surgery will give excellent clinical outcomes with minimal complications.

Contributors: SM wrote the manuscript and conceptualized the work; PA and HPS were responsible for revising it critically. SM will act as a study guarantor. All authors approved the final version of this manuscript and are responsible for all aspects of this study.

Funding: None; Competing interests: None stated.

\section{References}

1. Schultz E, Sapan MR, McHeffey-Atkinson B, Naidich JB, Arlen M. Case report 872. "Ancient" schwannoma (degenerated neurilemmoma). Skeletal Radiol. 1994;23:593-595.

2. Stout AP. Tumors of the Peripheral Nervous System. In: Atlas of Tumor Pathology, Armed Forces Institute of Pathology, Washington DC, Section, 1949.

3. Kransdorf MJ. Benign soft tissue tumours in large referral population: distribution of specific diagnosis by age sex and location. AJR am J Roentenol. 1995;164:395.

4. Enzinger FM, Weiss SW. Malignant tumours of peripheral nerves. In: Enzinger FM, Weiss SW, eds. Soft tissue tumors, vol.31. St. Louis, MO: Mosby Company, 2001:1209-1263.

5. Knight DMA, Brich R, Pringle J. Benign solitary schwannomas: a review of 234 cases, The Journal of Bone and Joint Surgery. 2007;89:382-387. 
6. Birch R, Bonney G, Wynn Parry CB. The peripheral nervous system and neoplastic disease. In: Surgical disorder of peripheral nerves. Edinburg: Churchill Livingstone. 1998;335-352.

7. Gosk J, Gutkowska O, Urban M, Wnukiewicz W, Reichert P, Ziółkowski P. Results of surgical treatment of schwannomas arising from extremities. Biomed Res Int. 2015;2015:547926.

8. Jenkins S. Solitary tumours of peripheral nerve trunks. J Bone Joint surg. 1952;34B:401-411.

9. Eroglu U, Bozkurt M, Ozates O, Akturk S, Tuna H.
Sciatic nerve schwannoma: case report. Turk Neurosurg. 2014;24:120-122.

10. Rajasekaran RB, Shanmuganathan R. Schwannoma of the Posterior Tibial Nerve Presenting as Tarsal Tunnel Syndrome: A Case Report with Emphasis on the Role of Microscope during Surgery. Case Rep Orthop. 2018; 2018:4704362.

11. Kim SM, Seo SW, Lee JY, Sung KS. Surgical outcome of schwannomas arising from major peripheral nerves in the lower limb. International Orthopaedics. 2012; 36:1721-1725. 